\title{
Design of Online Education Resource Scheduling Sys- tem Based on Multi-level SDN Architecture
}

\author{
TANG Li ${ }^{1}$,TANG Li $\mathrm{Li}^{2}$ \\ \{TangLi3812@163.com¹, TangLiLi3812@163.com²\} \\ (1. Jiangxi Institute of Applied Science and Technology,Nanchang 330100,China; \\ 2.Jiangxi Institute of Applied Science and Technology,Nanchang 330100,China)
}

\begin{abstract}
In order to improve the time efficiency of online education resource scheduling system in resource scheduling,an online education resource scheduling system based on a multi-level SDN architecture is designed.The hardware part of the system designed this time mainly includes a controller and an expansion board; the software of the system In part,the online education resource scheduling based on the multi-level SDN architecture is realized through the construction of the online education resource database and the extraction of the associated dimension features of the resource information flow.The experimental results show that the online education resource scheduling based on the multilevel SDN architecture is designed The system spends less time on resource scheduling than traditional systems, which proves that the system designed this time is more superior.
\end{abstract}

Keywords:multi-level SDN architecture;online education;resources;scheduling; equilibrium;

\section{Introduction}

In the process of global education information, streaming media technology has been widely used in the field of education. Modern distance education system based on network provides abundant information resources, friendly interaction performance and excellent openness. The transmission of multimedia files on the network has become an important technical problem in modern distance education system. The small size of streaming media, less resource consumption, short waiting time, multiple media types, low purchase cost and various compression rates provide effective solutions for modern distance education system. With the development of online education, the scale of online education data has become larger and larger, and the real-time requirement of online education data processing is higher and higher. The traditional online education resource scheduling system has a poor real-time performance in resource scheduling. Educational resource scheduling system. SDN architecture is a new software-based network architecture. Layer and forwarding layer, as well as centralized network state control, to achieve transparent multilevel control network strategy of the underlying infrastructure. The programmability of the network provides automatic management and unified control functions for the network, and effectively solves the current problems. The problems of poor flexibility, limited expansion of resources and diversification 
of service types in networking. The system designed in this paper completes the design of online educational resource scheduling system based on multi-layer SDN architecture from hardware and software aspects. Practice has proved that the real-time system based on multilayer SDN architecture designed in this paper has better real-time performance than the traditional system.

\section{Online Education Resource Scheduling System Framework}

The system designed this time is mainly divided into three layers,the system layer,the application layer,and the data layer.It also includes an administrator login interface,teaching material management interface,and member management interface. The main function of the administrator login interface is to enter the user name and password.Judgment,can modify,add and delete the content of the system;teaching material management interface,which mainly manages the content of tutoring materials,all materials can be displayed in full,and can also be displayed by type;member management interface,administrators can delete,modify and lock users, and can retrieve passwords for users who have forgotten their passwords. The framework of the online education resource scheduling system based on the multi-layer SDN architecture designed this time is shown in Figure 1:

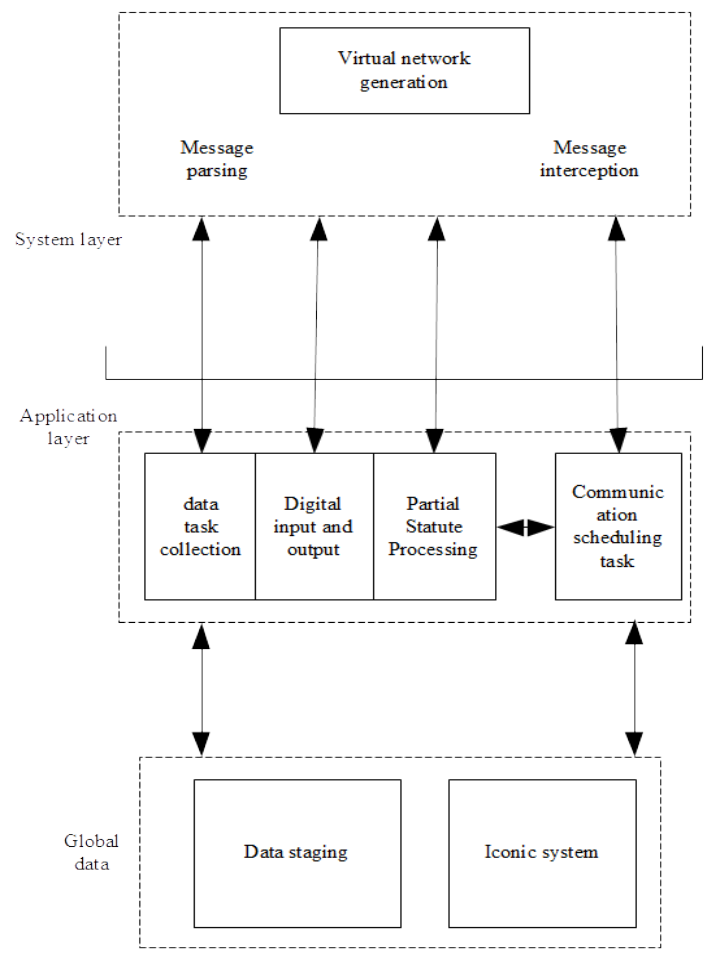

Fig.1.The framework of an online education resource scheduling system based on a multi-level SDN 


\section{architecture}

\section{Hardware design of online education resource scheduling system}

\subsection{Controller design}

The controller hardware can be divided into two parts:one is the USB control chip,and the other is the JTAG main control chip ${ }^{[1]}$.The controller uses the USB control chip to implement the USB protocol to communicate with the PC.At the same time,by operating the JTAG main control chip, The data output on the JTAG bus conforms to the IEEE1149.1 standard,so as to achieve the purpose of controlling the JTAG bus by a PC.The EZ-USBFX2 chip CY7C68013 produced by the company is a USB interface control chip,and the CY7C68013 chip includes an enhanced 8051 processing Device, a serial port engine(SIE), a USB2.0 transceiver, $8.5 \mathrm{kB}$ on-chip RAM,4kB FIFOMemoryAnd a universal programmable interface(gpif).Its gpif can be connected with any asic or dsp,it also supports all current universal bus standards. The USB interface chip ${ }^{[2]}$ of this system is used as an intermediary for data transmission, and it can be completed simultaneously with a portable computer. The exchange of control information and the execution of the control process. Transmission mode:Among the 4 transmission modes defined by the USB protocol,batch transmission and isochronous transmission are faster.However,batch transmission has error checking, which can ensure data transmission.Correctness.In this solution,because of the high requirements for the accuracy of the data, the batch transmission method is used.The 8051 running code is downloaded from the PC terminal.This way the system software modification and function upgrade are more flexible,and the external connection is also eliminated.rom,making the circuit more concise and reliable.

Select the company's boundary scan chip. The internal structure of the chip includes queue management module, host module, serial module, event manager, counter, command management and read-write bus.

\subsection{Expansion board design}

The w5100 network expansion board ${ }^{[3]}$ is used to provide communication functions for the innovative education network ecosystem. The expansion board integrates a 10/100 Ethernet controller to meet the system design requirements.

This chip supports hardware TCP/IP protocol,embedded 10 Base/100 BaseTX Ethernet physical layer ${ }^{[3]}$,and supports auto answer,ADSL link,4 independent ports,internal $16 \mathrm{~KB}$ memory as TX/RX cache,working The voltage is $3.3 \mathrm{~V}$, and the $\mathrm{I} / \mathrm{O}$ port can withstand $5 \mathrm{~V}$ voltage.At the same time,it contains a variety of indicator lights, where L represents the programming indicator;LINK represents the network has been linked,this indicator is flashing when sending and receiving innovative education network data; RX will flash when the 
network is receiving data;TX light will flash when the network detects a collision.

\section{3 processor design}

ADSP-21161N is a powerful 32-bit floating-point DSP chip recently launched by ADI of the United States.It uses a super Harvard structure,has multiple internal buses ${ }^{[4]}$,high-speed computing units,large-capacity memory,and flexible and diverse external interfaces.The core operating frequency can reach $100 \mathrm{MHz}$, and the external bus operating frequency can reach $50 \mathrm{MHz}$.Since it includes two sets of processing units inside,each set uses a three-stage pipeline structure for processing,so the operation processing speed can reach 600MIPS to achieve the DSP The function of low operating frequency and high processing capacity can reduce power consumption.Large-capacity internal dual-port SRAM,the capacity can reach $1 \mathrm{Mbit}$,divided into two storage areas,and one cycle can complete the access of instruction code and operands at the same time,and can be arbitrarily set It can be 16-bit,32-bit or 48-bit word width,which is convenient for different applications. The host(HOST)and multi-processor interface do not need external circuits,relying on the on-chip bus arbitration logic and DMA controller support,it can be easily Forms a tightly coupled parallel bus/shared memory parallel system. The on-chip SDRAM controller can directly manage SDRAM,and multiple DSPs can coordinate and use SDRAM well,so thatAn integrated processing system. Two sets of two-way high-speed LINK data transmission,each LINK port is supported by an independent DMA controller and send/receive data FIFO, which can perform high-speed data transmission up to $100 \mathrm{MB} / \mathrm{s}$,greatly improving Parallel processing capabilities can be used to form a loosely coupled distributed parallel system ${ }^{[5]}$.In addition,there are communication ports such as SPI interface,programmable I/O pins(FLAG), and synchronous serial ports.

\section{4 software design of online education resource scheduling system \\ 4.1 Construction of Online Education Resources Database}

Construct an online education resource scheduling database to reduce the time for online education resource scheduling. The distance education system includes 8 entity classes,student entity class,teacher entity class,system administrator entity class,course subject entity class,role entity class, test questions The entity class and the problem entity class are as follows.First,the student entity class,attributes:student number,user name,password,real name,class,mailbox,landline,address,mobile phone,etc. The student entity attribute diagram is shown in Figure 2: 


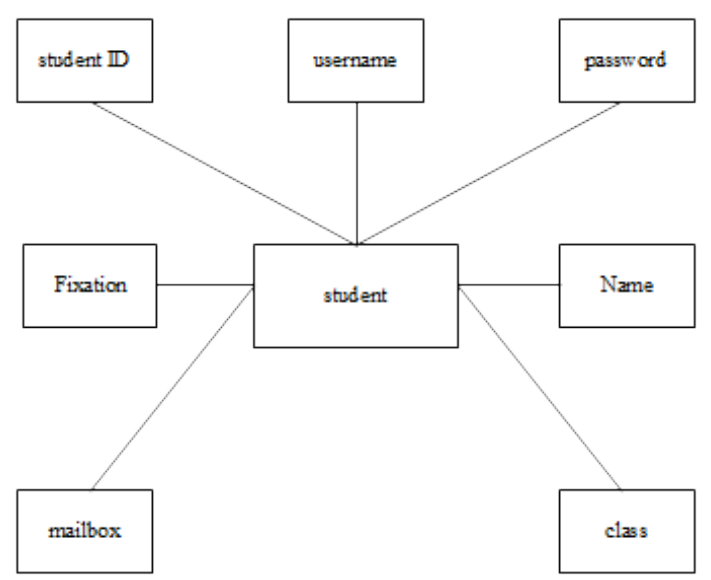

Fig. 2. Student entity attribute map

Second,the teacher entity class,attributes:work number,user name,password, real name, class, mailbox, fixed phone, address, mobile phone, etc.The attributes of the teacher entity are shown in Figure 3:

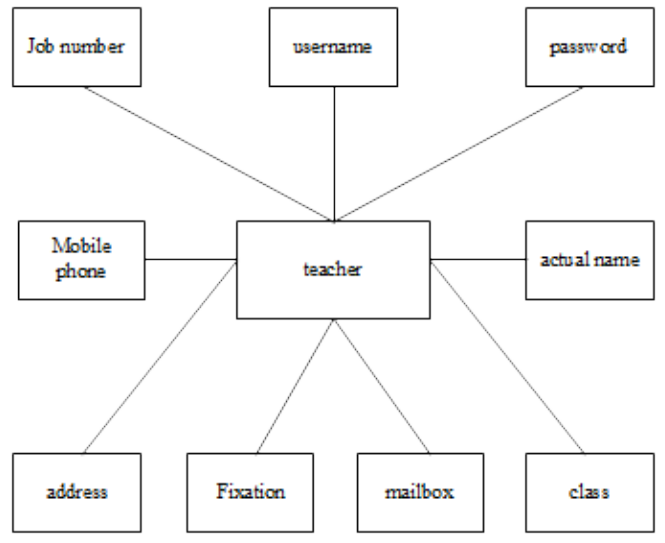

Fig.3. Teacher entity attribute map

Third, the system administrator entity class, attributes:number, user name, password, real name, mailbox, fixed phone, address, mobile phone, etc.The system administrator's implementation attribute diagram is shown in Figure 4: 


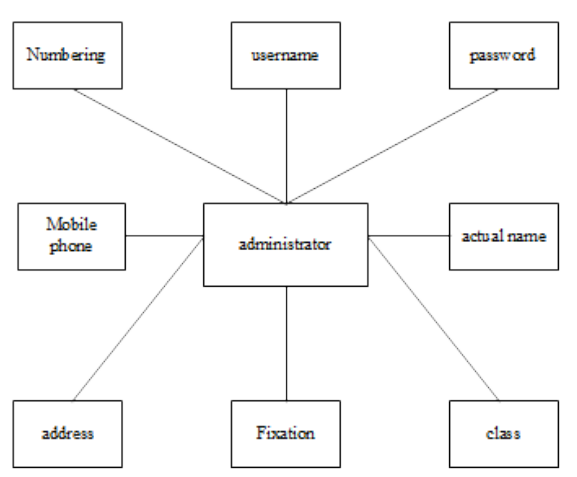

Fig.4. System administrator entity attribute map

Fourth:Course subject entity class,attributes,number,course subject name,etc.The course subject entity attributes are shown below:

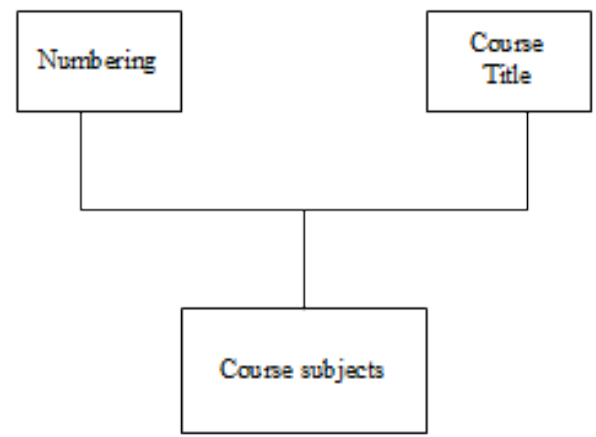

Fig.5. Course entity attribute map

Fifth:role entity class,attributes:number,role name,etc.The role entity attribute diagram is shown below:

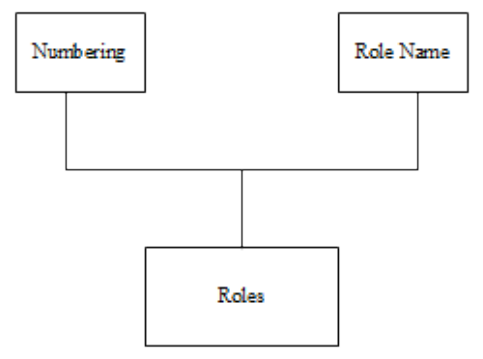

Fig.6. Character entity attribute map

Sixth,the entity type of the test question,attributes:number,title,answer option a,answer option b,answer option c,answer option d,correct answer.The test entity attribute diagram is 
shown in Figure 7:

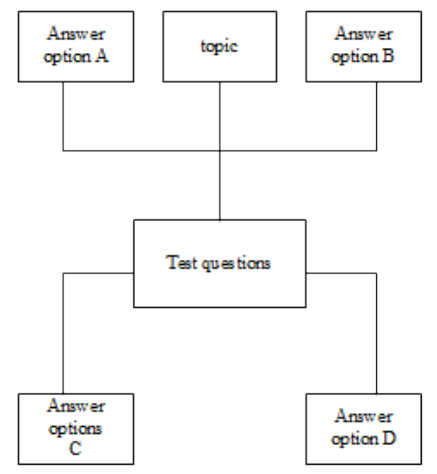

Fig. 7. Test entity attribute map

Seventh,the problem entity class,attributes:number,question title,answer analysis,etc. The problem entity attribute diagram is shown in Figure 8:

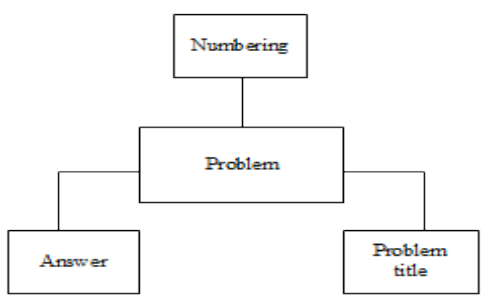

Fig. 8. Problem entity attribute map

The online education resource scheduling system stores education resources in accordance with the above database storage format to improve the speed of online education resource scheduling. The business flow chart of this database is shown in Figure 9: 


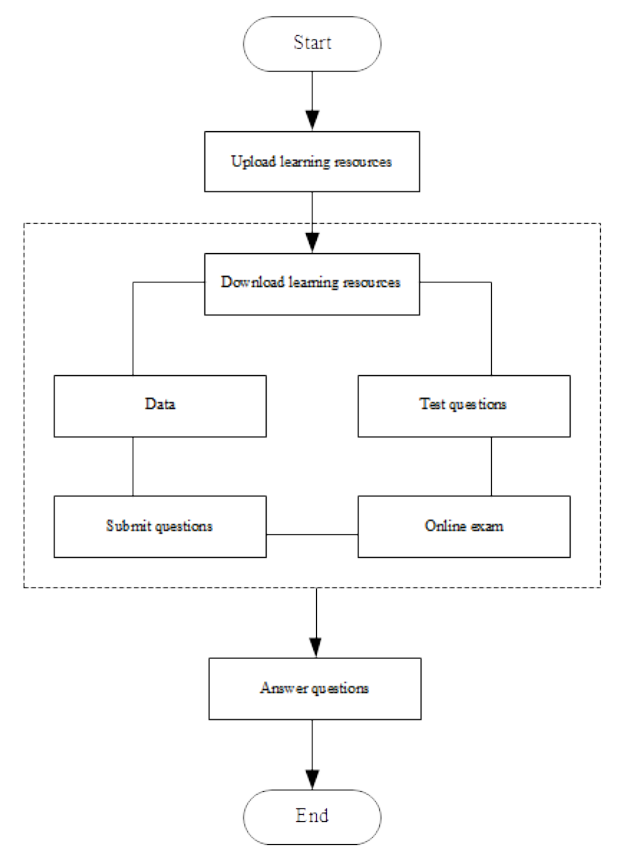

Fig. 9. Database business flow chart

\subsection{Relevant dimension feature extraction of resource information flow}

The traditional method uses the auto-correlation matching method of resource information for resource scheduling. When the interference in the data transmission channel is large and the prior data of the resource information flow is lacking, the balance ${ }^{[6]}$ of resource scheduling is not good,and the accuracy of registration is not good.In order to overcome the shortcomings of the traditional method,Gao designed an online education resource scheduling system based on a multi-level SDN architecture. The filter extracts the correlation dimension characteristics of the resource information flow, and firstly gives the function of information resource scheduling of open network large databases under cloud computing. The consumption is expressed as:

$$
F K=G \times G \frac{x}{M}(1)
$$

In formula(1), $F K$ represents a subspace ${ }^{[7]}$ the features of the associated dimension, $G$ represents resource filtering parameters, $G \frac{x}{M}$ represents power consumption parameters information resource scheduling.

Under the condition of resource load balancing,the cloud resource information is screened to achieve information fusion, and the association dimension single frequency 
characteristics of the open network large database information resources are obtained as follows:

$$
g_{f}=s d / \prod f
$$

In formula(2), $g_{f}$ represents real vector which the information resources of the open network large database, $s d$ is the information fusion scale in the correlation dimension feature extraction process, $\prod f$ is resource information flow.

Based on this, the correlation dimension features of the resource information flow are extracted.The graph extraction process is shown in Figure 10:

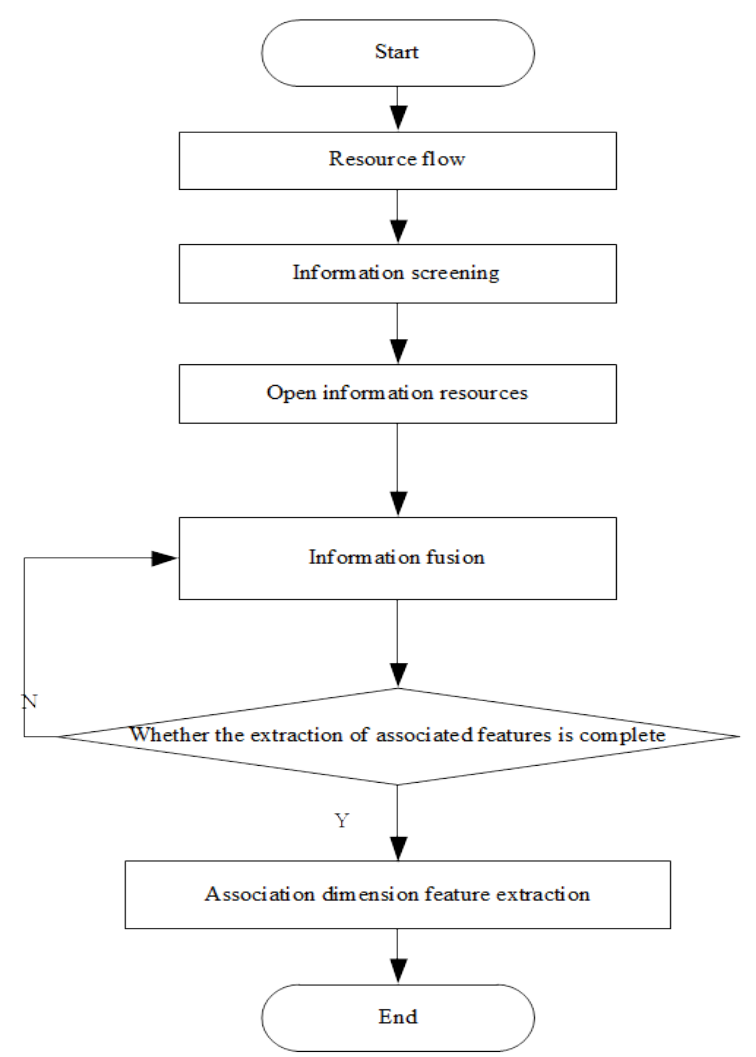

Fig. 10. Association dimension feature extraction process of resource information flow The calculation formula is: 


$$
|s|=\frac{d}{\sum a / g h}
$$

In formula(3), $|S|$ represents the time delay of resource scheduling, $\sum a$ is the scale factor, $g h$ is the spectral registration coefficient, $d$ is state transition parameters for resource scheduling.

As the time delay increases, the focus performance of the open network large database resource scheduling process improves.It can be seen that by extracting the associated dimension features of the resource information flow, the open network large database resource scheduling problem can be transformed into a multivariate unknown.Parametric channel adaptive equalization problem.

\subsection{Online Education Resource Scheduling}

According to the result of the feature extraction of the above resource information flow,resource load balancing control and channel adaptive equalization design are used to implement the open network large database resource scheduling.Under different interference intensities, the resource load balancing control function ${ }^{[8]}$ for resource scheduling is obtained as:

$$
T=\frac{a^{\prime \prime}}{s * d}(4)
$$

In formula(4), $T$ is the scale equilibrium coefficient, $S * d$ is scheduling the distribution elements for the open network large database resources, $a^{\prime \prime}$ is evenly distributed time window.

Based on the above calculations, the upper and lower thresholds of the resource scheduling of the open network large database are:

$$
F=\frac{|d|}{S * A}
$$

In formula(5), ${ }_{F}$ represents the information flow sampling window delay, $|d|$ represents the channel equalization coefficient, $S * A$ is continuous function vector.

Complete the online education resource scheduling based on the multi-layer SDN architecture according to the above process. The overall process is shown in Figure 11: 


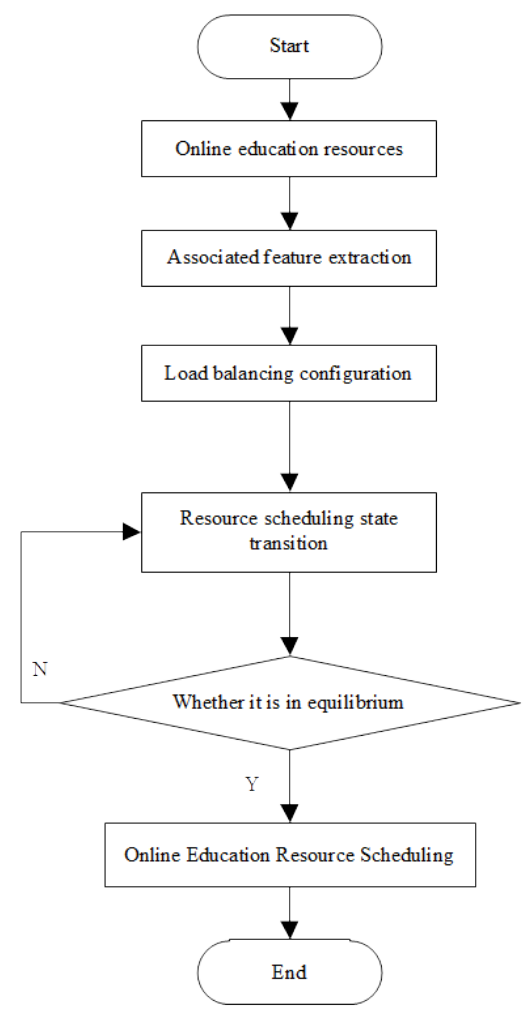

Fig. 11. Online education resource scheduling process

\section{Experimental comparison}

In order to verify the effectiveness of the multi-level SDN architecture-based online education resource scheduling system designed this time,an experimental comparison was performed.To ensure the rigor of the experiment,the traditional system was compared with the designed system,and the online education of the two systems was compared resource scheduling time.

\subsection{Experimental platform construction}

Set up a simulation platform based on Mininet. Mininet is a lightweight software-defined network test platform that can simulate a complete network host,link and switch on the same computer.The simulation platform supports custom complex topologies and can be on the same computer a complete network host,link and switch are simulated on the plane.The simulation platform supports custom complex topology,and the experimental platform is shown in Figure 12: 


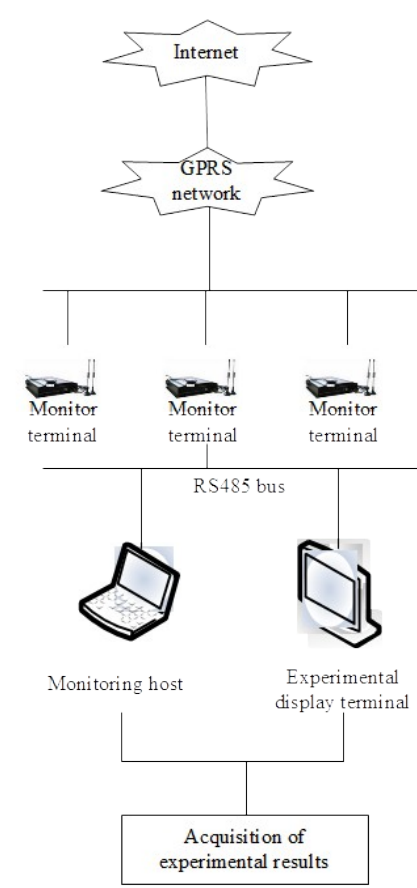

Fig. 12. Experimental platform

Use the above experimental platform for experiments.During the experiment,an online education platform was selected as the experimental object,and 7 experiments were performed,and the scheduling time of online education resources was compared with the 7 experimental processes.

\subsection{Analysis of experimental results}

The comparison result of traditional online education resource scheduling system based on multi-layer SDN architecture and traditional system online education resource scheduling time is shown in Figure 13: 


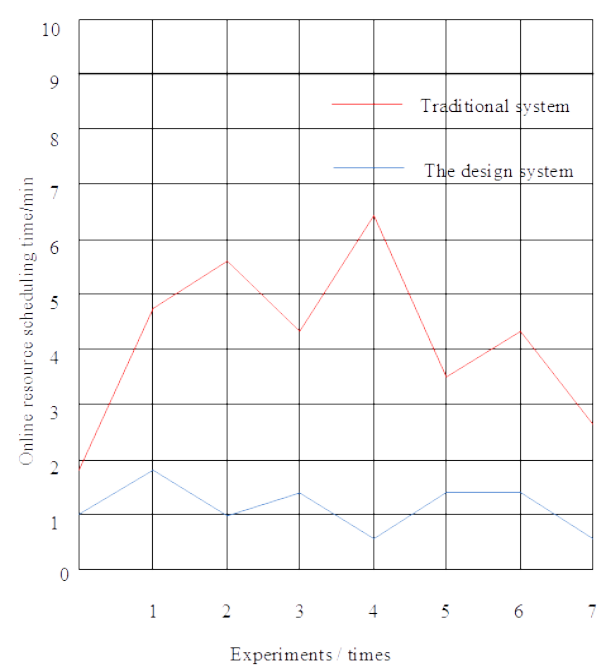

Fig. 13. Experimental comparison results

Based on the analysis of the above experimental comparison results, it can be seen that the online education resource scheduling time of the system designed this time is shorter,and the overall scheduling time is less than that of the traditional scheduling system.The traditional online education resource scheduling system has a longer scheduling time,which In the second experiment,the scheduling time was longer than that of the system designed this time.Therefore,the above experiments can prove that the system designed this time can reduce the scheduling time of online education resources and improve the real-time performance of online education resource scheduling system.

\section{Conclusion}

An online education resource scheduling system based on a multi-level SDN architecture is designed to solve the problem of long time for online education resource scheduling in traditional systems.It proves that the designed online education resource scheduling system improves the information registration ability of resource scheduling The overhead is small,the technical indicators are superior,and have high application value.There are still some imperfections in this design.If you design the parallel scheduling algorithm of the teaching resource access scheduling system,then combine linear programming and numerical values.Analyzing the theory,the effect will be better.We hope that the online education resource scheduling system based on the multi-layer SDN architecture designed this time can provide some help for other online education resource scheduling.

\section{References}

[1]Fei Xu,Wang Shaochang,Yang Weixia.Cloud Resource Scheduling Algorithm Based on Game 
Theory[J].Computer Science,2019,46(1):295-299.

[2]Gao Feng.Scheduling Algorithm Based on Improved Cloud Platform Data Center Resource[J].Bulletin of Science and Technology,2018,34(8):168-171.

[3]Zhen Yan,Zhao Hu.Resource scheduling strategy in hierarchical software defined wireless sensor networks[J].Journal of Xidian University(Natural Science),2019,46(4):87-98.

[4]Yan Jiankang,Chen Gengsheng.Improved H-Storm Platform Based on Co-scheduling of CPU/GPU Heterogeneous Resource[J].Computer Engineering,2018,44(4):1-11.

[5]Zheng Benli,Li Yuehui.Study on SDN Network Load Balancing Based on IACO[J].Computer Science,2019,46(1):291-294.

[6]Dou Haoming,Jiang Hui,Chen Siguang.SDN-based Network Controller Algorithm for Load Balancing[J].Computer Science,2019,46(6):312-316.

[7]Wang Ruyan,Zhou Jing,Wu Dapeng.A downlink resource scheduling strategy for C-RAN backhaul network[J].Journal of University of Science and Technology Beijing,2018,40(5):629-638.

[8]Bi Jingmei.On the Current Situation and Improvement Strategies of Online Learning for Higher Vocational College Students[J].Vocational and Technical Education,2017,38(14):36-40. 